\title{
Aspectos claves relacionados con la gestión del e.Learning
}

Francisco Lupiáñez Villanueva

Universitat Oberta de Catalunya.

La introducción y uso de las Tecnologías de la Información y la Comunicación, especialmente Internet, en el ámbito de la salud, la medicina y los sistemas sanitarios enfrenta a todos los agentes e instituciones ante numerosos retos. La educación y la formación, como actividades claves tanto de la sociedad de la información como de los sistemas sanitarios, también se ven afectadas por este proceso de cambio. La finalidad de este artículo es identificar y analizar algunos de los aspectos claves relacionados con la introducción de las TIC e Internet en el ámbito de la educación médica continuada desde el punto de vista organizacional.

Esta visión nos permite combinar factores pedagógicos, tecnológicos y organizativos. Todos ellos necesarios a la hora de abordar las complejas interacciones que se producen en la implantación de un proyecto de e.Learning.

Palabras clave: Internet, Educación Médica Continuada, e.Learning, Sociedad de la Información, Management

\section{Correspondencia:}

F. Lupiáñez

Profesor de los Estudios de Ciencias de la Información y la Comunicación de la Universitat Oberta de Catalunya. Avd. Tibidabo 4708035 Barcelona (Spain)

Tel (+34) $932535715 \quad$ Fax (+34) 932110126

Email: flupianez@uoc.edu Blog:www.ictconsequences.net

\section{INTERNET, TECNOLOGÍAS DE LA INFORMACIÓN Y LA COMUNICACIÓN Y SALUD}

Todas las sociedades son sociedades del conocimiento ya que ha sido este el recurso fundamental en la organización del poder, la riqueza, y la calidad de vida en cualquier época. Lo que es específico de nuestra sociedad no es que sea del conocimiento, sino el hecho de que en la sociedad actual la producción y generación de conocimiento y el procesamiento de información tiene una base tecnológica de nuevo tipo que permite que esa información y ese conocimiento sean difundidos y procesados en tiempo real a escala planetaria en el conjunto de los procesos que constituyen la actividad humana ${ }^{1}$.

La relación entre el contexto actual de la sociedad de la información, la medicina y los sistemas sanitarios es especialmente importante dado que en este ámbito de actividad la información y el conocimiento son especialmente relevantes.

Según Nettleton (2004), los cambios sociales, económicos y tecnológicos que se están produciendo en nuestra época plantean un cambio sustancial en los flujos de información y conocimiento que está reconfigurando los roles profesionales e institucionales, las prácticas médicas, la percepción del enfermo y la pro- 
pia conceptualización de la enfermedad. En este sentido, las innovaciones tecnológicas, especialmente las relacionadas con las TIC, sugieren que la agenda de actuación en los sistemas sanitarios esta siendo cada vez más "informacional" ${ }^{3}$.

Dentro de este nuevo contexto social, económico y tecnológico no es de extrañar que haya surgido conceptos como la eSalud (eHealth), para abarcar las posibles aplicaciones de las TIC, especialmente Internet, en el campo de la salud ${ }^{4}$. Una de estas aplicaciones es el uso de estas nuevas tecnologías para abordar las cuestiones relacionadas con la formación (eAprendizaje - e.Learning), cuestión clave dentro de los sistemas sanitarios.

\section{INTERNET, EDUCACIÓN MÉDICA Y e.LEARNING}

Si la introducción de las TIC en la salud esta produciendo cambios que nos hacen repensar la concepción de la propia medicina (Nettelton \& Burrows, 2003), también nos ha de hacer reflexionar sobre los cambios que se están produciendo en el campo de la educación médica ${ }^{5}$.

El uso de las TIC en la educación médica, especialmente Internet como medio de información, comunicación y organización social, ofrece nuevos escenarios y retos $^{6}$ a todos los profesionales. El e.Learning, entendido como el uso de las TICs para ofrecer una abanico amplio de soluciones que aumenten los conocimientos y el rendimiento de los profesionales $^{7}$, ha de superar, como en cualquier proceso de cambio/crisis, numerosos mitos ${ }^{8}$.

Existe ya evidencia que destaca que el uso de Internet para la educación medica es tan efectivo como los medios que tradicionalmente se han utilizado $^{9}$. No obstante, a pesar de esta efectividad y de los niveles de satisfacción alcanzado ${ }^{10}$ no se deben de dejar de lado la existencia de numerosas barreras y fricciones relacionadas con factores organizativos, culturales y sociales propias de la introducción de este tipo de tecnologías en los contextos sanitarios.

\section{ASPECTOS CLAVE DEL E.LEARNING ${ }^{\text {a PARA }}$ LA EDUCACIÓN MÉDICA}

Para la introducción y el uso del e.Learning en la educación médica hemos de tener en cuenta factores relacionados con aspectos organizativos, educativos y tecnológicos. Es en la intersección de estas tres esferas ${ }^{11}$ desde donde se podrán afrontar los complejas interacciones que supone la introducción y uso de una nueva tecnología en un "antigua" actividad como es la educación. Tradicionalmente se han prestado atención a los aspectos pedagógicos y tecnológicos, no obstante la perspectiva organizacional también es clave para afrontar con este proceso.

\section{- Nueva actividad}

Antes de iniciar cualquier actividad formativa con un uso de tecnologías es condición necesaria definir el "producto" que vamos a ofrecer, teniendo en cuenta los destinatarios finales, y pensar el por qué necesitamos utilizar la tecnología para realizar esa oferta. Este tipo de cuestiones nos darán una primera aproximación de la envergadura del proyecto y colocará la tecnología alineada con la finalidad del mismo.

El abanico de soluciones que permiten los avances tecnológicos estará siempre al servicio de las finalidades del proyecto y enmarcado dentro del contexto social donde se desarrollará la actividad formativa. En este sentido, Internet permite abordar las variables del tiempo y del espacio de diferentes maneras, lo que facilita una gran cantidad de opciones que van desde la total virtualidad de la acción, a acciones mixtas (blended learning) hasta la total presencialidad del proceso utilizando Internet para cuestiones puntuales.

En toda esta planificación de la nueva actividad hemos de tener en cuenta los posibles competidores que existen tanto en el contexto donde se vaya a desarrollar la acción formativa como en el mercado. De esta manera podremos identificar la complementariedad con otros productos o acciones y buscar sinergias o detectar posibles conflictos. Toda esta reflexión

a. La idea original de los aspectos clave del e.Learning fue desarrollada inicialmente por Xavier Aragay y Carles Esquerré fruto de su experiencia como Gerente y Gerente Adjunto, respectivamente, de la Universitat Oberta de Catalunya. 
nos facilitará analizar los factores clave que permitirán que nuestra actividad sea exitosa.

\section{- Gobernabilidad}

Dependiendo de la envergadura de nuestro proyecto tendremos que tener en cuenta cuestiones relacionadas con los diferentes grupos de interés que estarán involucrados. Imaginemos que el proyecto que estamos configurando afecta a los profesionales sanitarios de toda una región, tendremos entonces que prestar especial atención a las fuerzas políticas y sindicales. Analizar que posición adoptan ante nuestro proyecto.

Imaginémonos que el proyecto abarca la relación entre varios departamentos de diferentes hospitales que participan en un proyecto de investigación. Tendremos entonces que tener en cuenta el poder de cada departamento dentro de cada hospital y las personas claves de cada institución.

Esta análisis nos permitirá detectar a los posibles aliados o impulsores de nuestra iniciativa, así como los posibles grupos de presión o freno.

Otra cuestión directamente relacionada con la gobernabilidad del proyecto es la formula legal y los órganos de gobierno seleccionados. Hemos de tener en cuenta este tipo de cuestiones estructurales antes de comenzar el proyecto ya que nos permitirán posicionar nuestra iniciativa dentro del contexto organizativo y social donde se desarrolle. En este sentido es fundamental tener en cuenta la capacidad de liderazgo e influencia que tenga la persona responsable del proyecto.

\section{- Estructura organizativa y viabilidad económica del proyecto}

Este punto está estrechamente relacionado con los aspectos relacionados con la gobernablidad del proyecto.

Llevar a cabo las acciones asociadas a un proyecto de e.Learning requiere de una cierta estructura organizativa que dependerá fundamentalmente de la envergadura del proyecto. Esta estructura habrá de tener una dirección capaz de impulsar y coordinar el proyecto con un liderazgo destacado. Tendrán que definirse funciones y procesos de trabajo que acoten la responsabilidad sobre las diferentes actuaciones que han de desarrollarse, teniendo en cuenta una cultura organizativa orientada a resultados y donde se valore la innovación y la capacidad de trabajar en equipo.

Para todo ello es necesario el diseño de una plantilla de profesionales (informáticos, pedagogos, expertos en comunicación, profesionales sanitarios,...) con un perfil definido que tengan los incentivos adecuados para mantener la motivación y el entusiasmo en el proyecto.

Obviamente todo esto tendrá que estar acompañado de una previsión de ingresos y gastos así como de un plan de inversiones y de financiación.

\section{- Modelo pedagógico}

La reflexión realizada con la definición de la nueva actividad que vamos a emprender ha de materializarse en un modelo que recoja todos los aspectos relacionados con la parte pedagógica de la acción formativa.

El rol del estudiante, el rol del profesor, facilitador o tutor, el rol de los materiales educativos, la plataforma tecnológica, la biblioteca, el método de evaluación y la definición de las variables relacionadas con el tiempo (sincronía - asincronía) y el espacio (totalmente virtual - totalmente presencial) son algunos de los elementos que tendrá que recoger nuestro modelo pedagógio.

De nuevo hemos de recalcar la importancia de que el modelo definido este al servicio de la finalidad que queremos alcanzar teniendo presentes las variables relacionadas con el contexto tanto interno como externo.

Si decidimos que la acción formativa será totalmente virtual y que coincidirá en el tiempo (sincronía) utilizaremos herramientas que permitan la interacción de los agentes en tiempo real (videoconferencia o sistemas de mensajería instantanea) y un tipo de materiales educativos determinados. En cambio si nuestro proyecto opta por un sistema totalmente virtual pero asíncrono (no coinciden en el tiempo), haremos un mayor uso de otro tipo de herramientas como foros o el correo electrónico. Quizá debemos exigir entonces un tiempo mínimo de respuesta a estos mensajes y por lo tanto un rol del profesor determinado. Esto también condicionará la selección o creación de los materiales educativos e incluso el perfil del estudiante.

Las combinaciones son casi infinitas de ahí la importancia de una buena definición del proyecto que nos facilite concebir un modelo pedagógico con los elementos necesarios $\mathrm{y}$, sobre todo, con una coherencia entre ellos.

\section{- Sistema de información y gestión académica}

Una vez resueltas las cuestiones relacionadas con el modelo pedagógico que vamos a establecer es 
fundamental dotarlo de una arquitectura tecnológica apropiada y de una gestión óptima.

Existen un sinfín de siglas ${ }^{\mathrm{b}}$ relacionadas con las aplicaciones que permiten la acción formativa mediada por las TIC y al menos tres posibilidades:

- Adquirir un software ya desarrollado

en el mercado.

- Desarrollar aplicaciones dentro del propio proyecto.

- Adaptar soluciones de código abierto (software libre)

Estamos ante una cuestión importante, no obstante no hemos de olvidar, sin dejar de lado la gestión y el mantenimiento de esta tecnología, que la finalidad del proyecto es el punto clave y por tanto la solución tecnológica que se adopte ha de estar perfectamente alineada con esta finalidad.

Un condicionante importante de esta decisión está relacionado con el ordenador de trabajo del destinatario final de la acción formativa y la calidad de conectividad a la Red que posea. Este tipo de cuestiones también han de ser coherentes con los materiales educativos o las aplicaciones necesarias para que el estudiante alcance sus objetivos de aprendizaje.

Por último, hemos de tener en cuenta las cuestiones relacionadas con la gestión de la acción formativa (matriculas, pagos, secretaria académica, gestión de la creación de materiales...) y si nuestros sistemas de información soportaran o no esta gestión. De nuevo hemos de volver la vista atrás hacia la definición del proyecto que hayamos hecho. Es posible que la gestión académica sea realizada de manera presencial o mediante otro tipo de sistemas de información no incluidos en nuestras aplicaciones informáticas. $\mathrm{O}$ puede ser imprescindible que este tipo de gestiones también se realicen on-line y estén perfectamente integradas con el resto de sistemas. Incluso puede tratarse una acción formativa no reglada que no requiere de este tipo de procesos en cambio sea necesario que facilite la creación de contenidos online para los estudiantes.

\section{- Comunicación y servicios}

Además de las cuestiones relacionadas con el marketing que tienen que ver con el análisis de la

b. Learning Management System (LMS), Course Management System (CMS), Learning Content Management System (LCMS), Managed Learning Environment (MLE), Learning Support System (LSS) or Learning Platform (LP). oferta y la demanda es importante tener en cuenta una política de información y comunicación que nos facilite atraer, mantener y fidelizar a todos los participantes de la acción formativa.

No hemos de olvidar que estamos ante un nuevo espacio mediado por ordenadores que permite en muchos casos reproducir y distribuir nuestra "imagen" con gran facilidad. Esto nos enfrenta a nuevos retos y posibilidades pero también a riesgos.

Unas normas de buena conducta en los espacios virtuales comunes, unas guías que faciliten la toma de contacto con el nuevo entorno a los participantes, un política que recoja la pro activad por parte de los profesores ante la ausencia de mensajes del estudiante... son algunas de las cuestiones a las que deberían prestarse atención.

Además de las acciones relacionadas directamente con la acción formativa es necesario prestar atención a otro tipo de servicios que se puedan ofrecer tanto a través de la Red como presencialmente, en función de la definición del proyecto.

Los aspectos relacionados con acciones que permitan que los estudiantes compartan espacios más allá de la actividad formativa permiten generar vínculos emocionales que además de facilitar el aprendizaje generan sinergias positivas. Creación de foros por parte de los estudiantes en torno a temáticas diversas, posibilidad de que los estudiantes tengan un espacio propio en la Red, encuentros presenciales... son tan solo algunas de las posibilidades que favorecen la creación y la sensación de pertenencia a una comunidad ya sea totalmente virtual o mixta.

\section{- Diseño de la experiencia piloto}

Por último, es importante diseñar una experiencia piloto con un número reducido de participantes que nos permita evaluar el proyecto antes de su puesta en marcha definitiva. En este proceso se pueden identificar aspectos que no hemos tenido en cuenta o detectar problemas de funcionamiento que nos permitirán comenzar el proyecto con más probabilidades de éxito.

\section{CONCLUSIONES}

La medicina y los sistemas sanitarios tienen en la introducción y uso de las TICs, especialmente Internet, un reto que afecta a todas las actividades, agentes y organizaciones de este sector. Obviamente, la educación médica no esta excluida de este proceso. 
La introducción y el uso de las tecnologías de Internet en este ámbito ha de tener en cuenta factores relacionados con la tecnología, la pedagogía y la organización. Si se ha recalcado que la tecnología no es un fin en si misma, hemos de pensar que tampoco lo son ni la pedagogía ni la organización. Es en la intersección de estas tres esferas donde se encuentran las claves para la introducción de Internet, como medio de información, comunicación y organización social, en la educación médica.

La reflexión sobre la nueva actividad que vamos a impulsar; los aspectos relacionados con la gobernabilidad y la estructura organizativa de nuestro proyecto; el modelo pedagógico que vamos a implementar; los sistemas de información y gestión necesarios; la atención a la comunicación y los servicios que ofreceremos y, por último, el diseño de una prueba piloto son factores a tener en cuenta antes de sumergirse en un proyecto de e.Learning.

\section{BIBLIOGRAFÍA}

1. Castells, M. (2000). La era de la información. La sociedad red. Vol. 1. Madrid. Alianza.

2. Nettleton, S. (2004). "The Emergence of E-Scaped Medicine?" Sociology 38(4): 661 - 679.

3. Webster, A. (2002). "Innovative Health Technologies and the Social: Redefining Health, Medicine and Body." Current
Sociology 50(3): 443-457.

4. Pagliari, C., et al. (2005). "What is eHealth (4): A Scoping Exercise to Map the Field." Journal of Medical Internet Research 7 (1): 14.

5. Manning, P. R., DeBakey, L. (2001). "Continuing Medical Education: The paradigm is changing." The Journal of Continuing Education in the Health Professions 21(1): 46-54.

6. Harden, R. M. (2002). "Myths and e.Learning." Medical Teacher 24(5): 469-472.

7. Ruiz, J. G., Mintzer, M.J., Leipzig, R.M., (2006). “The Impact of E-learing in Medical Education." Academic Medicine 81(3): 207-211.

8. Harden, R. M. (2005). "A New Vision For Distance Learning and Continuing Medical Education." The Journal of Continuing Education in the Health Professions 25(1): 43-51.

11. Wutoh, R., Boren, S.A., Balas, E.A. (2004). "e.Learning: A Review of Internet-Based Continuing Medical Education." The Journal of Continuing Education in the Health Professions 24(1): 20-30.

10. Cobb, S. C. (2004). "Internet Continuing Education for Health Care Professionals: An Integrative Review." The Journal of Continuing Education in the Health Professions 24(3): 171-180.

11. Duart, J.M.; Lupiáñez, F. (coords.). (2005). Las TIC en la universidad: estrategia y transformación institucional [monográfico en línea]. Revista de Universidad y Sociedad del Conocimiento (RUSC). Vol. 2, núm. 1. UOC.

[Fecha de consulta: dd/mm/aa].

<http://www.uoc.edu/rusc/dt/esp/conclusiones0405.pdf> 